${ }^{1}$ Institute for Safety Problems of Nuclear Power Plants, NAS of Ukraine, 36a, Kirova st., Chornobyl, 07270, Ukraine,

${ }^{2}$ State Specialized Enterprise “Chornobyl NPP”, Slavutych, 07101, Ukraine

\title{
Investigation of Radionuclide Contamination of Materials Stored in Solid Radwaste Repository of the Chornobyl NPP
}

Keywords:

Chornobyl NPP,

solid radioactive waste,

specific activities,

difficult-to-measure radionuclides,

key nuclides,

correlation factor,

scaling factor
The composition and features of radionuclide contamination of solid radioactive waste (SRW) stored in the western "light" compartment of SRW repository (RSRW) of the SSE "Chornobyl Nuclear Power Plant" (ChNPP) were studied. Representative samples of SRW taken from the top layer of materials at 1-m depth were split into four streams, and according to their physical state, they were classified into two categories: combustible (one stream) and non-combustible (three streams) ones. After relevant sample preparation was carried out, the SRW samples were investigated in the ChNPP Laboratory for water-radiochemical measurement in order to identify the radionuclides, which are subject to compulsory certification according to SRW acceptance criteria valid in the Exclusion Zone. It was stated that SRW radioactivity levels in the upper layer of western "light" compartment of SWR are, mainly, determined by radionuclide contents of ${ }^{137} \mathrm{Cs},{ }^{60} \mathrm{Co},{ }^{90} \mathrm{Sr},{ }^{94} \mathrm{Nb},{ }^{235} \mathrm{U},{ }^{238} \mathrm{U},{ }^{241} \mathrm{Am},{ }^{3} \mathrm{H}$ and ${ }^{14} \mathrm{C}$. The parameters of regression dependencies and correlation coefficients between the specific activity of radionuclides in stream- and integrated (for non-combustible SRW) data samples compiled of measurement results (above the minimally detectable activity) were estimated. It was stated that the features of radionuclide contamination spectrum of the SRW in western and eastern compartments are largely similar ones, that, apparently reflects the typical properties of the ChNPP operational wastes produced in period from 1978 to 1986, and which had been stored in mothballed state in the RSRW before the accident at the ChNPP Unit 4 occurred. However, for such radionuclides as ${ }^{60} \mathrm{Co},{ }^{94} \mathrm{Nb}$ and ${ }^{90} \mathrm{Sr}$, observable levels of SRW contamination in the western compartment are significantly higher, than in the eastern one. At the same time, the specific activity of uranium isotopes ${ }^{235,238} \mathrm{U}$ is several times lower. It was concluded that the experimental and analytical results obtained in this work can be used as the source data for the next stage of studies - calculation of scaling factors and determination of radionuclide vectors needed to certify the difficult-to-measure radionuclides in the ChNPP SRW packages being transported to their burial.

\section{Introduction}

In 2016, the preparatory works of the Chornobyl NPP (ChNPP) radwaste certification at the Industrial Complex for Solid Radioactive Waste (SRW) Management (ICSRWM) were started in the SRW. Based on the results of radiation monitoring, each SRW batch (package) sent to the burial shall receive a certificate with all indicated characteristics needed by waste acceptance Criteria [1], including specific and total activity of radiologically significant nuclides. However, the results of preliminary tests [2] have demonstrated that directly in the package, standard 
equipment of ICSRWM's radiation-technological monitoring system (RTMS) allows reliably measuring the specific activity of gamma-emitters only, such as ${ }^{60} \mathrm{Co}$ and ${ }^{137} \mathrm{Cs}$. To get out of this situation, the experts of WANO technical support mission proposed to determine the content of remaining alpha- and beta-emitting nuclides subject to certification using scaling factors (SF) methodology $[3,4]$. The procedure of SF values (radionuclide vector) establishment requires representative sampling of each separate batch of waste, statistical analysis of quantitative relationships between the activity of difficult-to-measure (DTM) and key nuclides (KN), and if needed, correlationregression analysis of measurement results $[5,6]$. A similar work was performed for the operational ChNPP SRW in eastern "light" compartment of SRW repository (RSRW), which was mothballed before the accident of the ChNPP Unit 4 in 1986 [7, 8]. The quantitative relationships between the radionuclide content in SRW contamination spectrum were established. For all the DTMs subject to declaration, the SF values were calculated, which allow evaluating certification activity of radionuclides according to gamma spectrometry results of $\mathrm{KN}$ content on RTMS equipment.

This work is aimed at the study of the ChNPP SRW stockpiled in the western "light" compartment of RSRW, and obtaining the needed data to characterize their radionuclide composition.

\section{Objects and methods}

The investigation was focused on the materials classified as SRW and stockpiled in the western "light" compartment of the ChNPP RSRW. This compartment was filled with the waste produced during the normal operation of the ChNPP power Units, or as a result of buildingand reconstruction works in the period from 1978 to 1986 (operational waste). The compartment was mothballed before the ChNPP Unit 4 accident in 1986. The total amount of waste accepted for storage made $668 \mathrm{~m}^{3}$ with average density being $1.5 \cdot 10^{3} \mathrm{~kg} / \mathrm{m}^{3}$.

To determine the content (specific activity) of alpha-, beta- and gamma-emitting radionuclides subject to certification in accordance with [1], 124 representative samples were taken. The sampling was carried out by the envelope method from 9 points (Fig. 1) from up to 1-m depth. After visual inspection, the material samples were split into four SRW streams (Table 1). The SRW samples were investigated in the ChNPP Laboratory for radiochemical water measurement (LRWM). Methods, techniques and characteristics of LRWM devices used to determine the radionuclide content (Table 2), are given in [7].

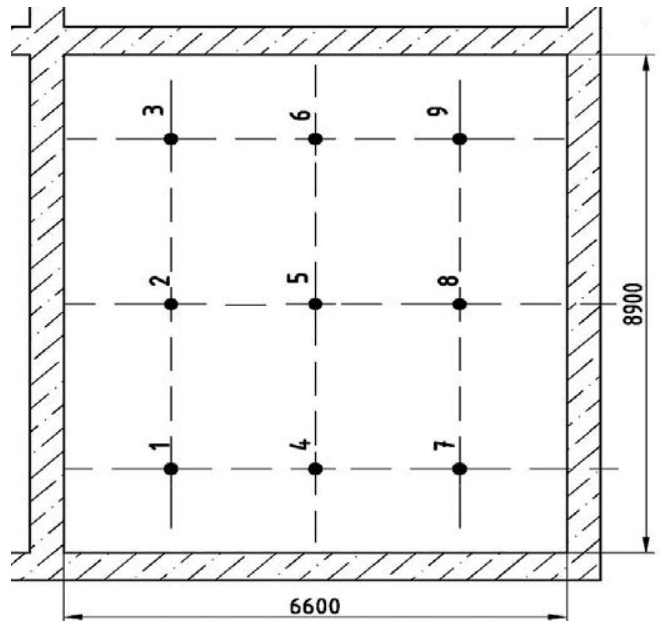

Fig. 1. Sampling scheme in the western "light" compartment of the ChNPP RSRW. Dimensions given in $\mathrm{mm}$

Table 1. General characterization of waste materials split into four streams

\begin{tabular}{|c|c|}
\hline Stream no. & Waste materials \\
\hline \multicolumn{2}{|c|}{ Non-combustible } \\
\hline 2 & $\begin{array}{c}\text { Metal products (impulse pipes of stainless steel } \\
\text { and equipment elements, abrasive discs) }\end{array}$ \\
\hline 3 & $\begin{array}{c}\text { Non-combustible cable products and heat } \\
\text { insulation unsuitable to reuse }\end{array}$ \\
\hline \multicolumn{2}{|c|}{ etc.) - products of recovery and repair works } \\
\hline 4 & $\begin{array}{c}\text { Wood, rubber, rags, worn special clothing, } \\
\text { spent personal protective equipment (PPE), } \\
\text { paper, polyethylene, organic materials (PVC) }\end{array}$ \\
\hline
\end{tabular}

Table 2. Methods and instrumentation used in the work

\begin{tabular}{|c|c|c|}
\hline $\begin{array}{l}\text { Measurement } \\
\text { method }\end{array}$ & Radionuclides & Devices \\
\hline $\begin{array}{l}\text { Gamma-ray } \\
\text { spectrometry }\end{array}$ & $\begin{array}{l}{ }^{60} \mathrm{Co},{ }^{94} \mathrm{Nb}, \\
{ }^{137} \mathrm{Cs},{ }^{241} \mathrm{Am}\end{array}$ & $\begin{array}{c}\text { HPGe gamma-ray } \\
\text { spectrometer SEG-002 } \\
\text { (“ATOMKOMPLEX- } \\
\text { PRIBOR”, Ukraine) }\end{array}$ \\
\hline $\begin{array}{l}\text { Liquid } \\
\text { scintillation } \\
\text { counting }\end{array}$ & $\begin{array}{l}{ }^{3} \mathrm{H}, \mathrm{C}^{14},{ }^{63} \mathrm{Ni} \\
{ }^{90} \mathrm{Sr},{ }^{241} \mathrm{Pu}\end{array}$ & $\begin{array}{l}\text { Liquid scintillation } \\
\text { counter Hidex 300SL }\end{array}$ \\
\hline $\begin{array}{l}\text { Alpha-particles } \\
\text { spectrometry }\end{array}$ & $\begin{array}{l}{ }^{237} \mathrm{~Np},{ }^{238} \mathrm{Pu}, \\
{ }^{241,243} \mathrm{Am}\end{array}$ & $\begin{array}{c}\text { Alpha-particles } \\
\text { spectrometer SEA-01 } \\
\text { (“ATOMKOMPLEX- } \\
\text { PRIBOR”, Ukraine) }\end{array}$ \\
\hline $\begin{array}{l}\text { ICP mass- } \\
\text { spectrometry }\end{array}$ & $\begin{array}{l}{ }^{10} \mathrm{Be},{ }^{59,},{ }^{63} \mathrm{Ni},{ }^{93} \mathrm{Zr}, \\
{ }^{94} \mathrm{Nb},{ }^{99} \mathrm{Tc},{ }^{129} \mathrm{I}, \\
{ }^{135} \mathrm{Cs},{ }^{237} \mathrm{~Np},{ }^{235}, \\
{ }^{236,238} \mathrm{U},{ }^{243} \mathrm{Am}, \\
{ }^{239,240,241,242} \mathrm{Pu}\end{array}$ & $\begin{array}{l}\text { Mass-spectrometry } \\
\text { system Agilent } 7500 \\
\text { Series ICP-MS }\end{array}$ \\
\hline
\end{tabular}


The parameters of radionuclides specific activity distribution, regression dependencies and correlation coefficients (CC) between radionuclide specific activity were estimated in stream- (stream sampling) datasets compiled of measurement results with detectable activity (above the minimally detectable activity - MDA). To improve statistical reliability of estimated parameters in non-combustible SRW and to compare with similar values obtained earlier for the eastern compartment [8], integrated datasets were also analyzed by way of combining the streaming samples (in a combination $1+2+3$ or only $2+3$ in the absence of significant results for stream no. 1). For the same purpose, the parameters of activity distribution and $\mathrm{CC}$ values were estimated for such pairs of radionuclides as ${ }^{137} \mathrm{Cs}-{ }^{60} \mathrm{Co},{ }^{235} \mathrm{U}-{ }^{238} \mathrm{U}$ and ${ }^{3} \mathrm{He}-{ }^{14} \mathrm{C}$.

All analyzed data samples were preliminarily checked for availability of abnormal results (according to Grubbs outliers test, [9]) for confidence level of $95 \%$. The check was carried out for the logarithm of radionuclide specific activity, which allowed minimizing erroneous exclusion of boundary values from the data spectrum of lognormally distributed radionuclides. The procedure for carrying out the correlation-regression analysis, testing the hypothesis for a linear relationship presence between the activity logarithms of type $\operatorname{Ln}\left(A_{D T M}\right)=\operatorname{Ln}(a)+b \cdot \operatorname{Ln}\left(A_{K N}\right)$ and the procedure for assessing $C C$ reliability, met the instructions of manual [10] and methodology described in [8].

\section{Results and Discussion}

Tables 3 and 4 show the features of radionuclide spectrum (for KN and DTM nuclides, respectively) and their specific activity measured within the RSW content from different streams. In the rows marked as " $1+2+3$ " or " $2+3$ ", the data for integrated samples (non-combustible SRW) are given.
As it can be seen, radioactive contamination of the studied SRW from RSRW western compartment is determined by several radionuclides only, and does not, practically, differ in this aspect from the SRW stockpiled in eastern compartment $[7,8]$. Specific activity of the rest radionuclides from those given in Table 2, and which are subject to certification according to the measurement results, did not exceed the MDA values of LRWM instrumentation shown in [7] (see Table 3 MDA values). From the Tables 3 and 4 data it is seen that the variation of specific activity values of radionuclides in waste materials is very significant and can reach five orders of magnitude $\left({ }^{60} \mathrm{Co},{ }^{94} \mathrm{Nb}\right)$ within a stream. As it is evidenced by the data $[5,6]$, this is a typical picture for the SRW producible at the NPPs. Therefore, when conducting statistical data analysis, the fact that the density of measurement results distribution in a representative sample is usually lognormal, is recommended to take into account. And this, in its turn, dictates the need in analyzing the distribution of not nuclide activity in the SRW, but its logarithm. This circumstance was considered within the framework of current work.

If we compare the measured levels of SRW radioactivity with similar data for the eastern compartment, the following trends can be noted:

specific activities of ${ }^{94} \mathrm{Nb}$ and ${ }^{60} \mathrm{Co}$ in the SRW streams in the western section are, generally, higher than in the eastern compartment. For some streams (No. 3 and 4) - within one, in others (No. 1 and 2) - by 1-2 orders of magnitude. The only exception is stream 3 , for which the specific activity of ${ }^{60} \mathrm{Co}$ in SRW was at, approximately, the same level of values, the both are in the range and in the average value;

a similar trend is observed for ${ }^{137} \mathrm{Cs},{ }^{241} \mathrm{Am}$ and ${ }^{90} \mathrm{Sr}$, but only for combustible SRW. For the rest SRW streams,

Table 3. Characteristics of specific activity of key radionuclides in SRW streams

\begin{tabular}{|c|c|c|c|c|c|}
\hline \multirow{2}{*}{ Radionuclides } & \multirow{2}{*}{ Stream No. } & \multirow{2}{*}{$\begin{array}{l}\text { Data amount } \\
(>\mathrm{MDA})^{*}\end{array}$} & \multicolumn{3}{|c|}{ Specific activity, $\mathrm{Bq} / \mathrm{kg}$} \\
\hline & & & minimum & maximum & average \\
\hline \multirow{5}{*}{${ }^{137} \mathrm{Cs}$} & 1 & $12(100)$ & $6.20 \cdot 10^{+2}$ & $5.80 \cdot 10^{+5}$ & $6.52 \cdot 10^{+4}$ \\
\hline & 2 & $31(100)$ & $4.11 \cdot 10^{+2}$ & $2.39 \cdot 10^{+5}$ & $4.39 \cdot 10^{+4}$ \\
\hline & 3 & $26(100)$ & $2.62 \cdot 10^{+2}$ & $8.89 \cdot 10^{+4}$ & $1.35 \cdot 10^{+4}$ \\
\hline & $1+2+3$ & $69(100)$ & $2.62 \cdot 10^{+2}$ & $5.80 \cdot 10^{+5}$ & $3.62 \cdot 10^{+4}$ \\
\hline & 4 & $55(100)$ & $1.84 \cdot 10^{+2}$ & $3.81 \cdot 10^{+6}$ & $2.62 \cdot 10^{+5}$ \\
\hline \multirow{5}{*}{${ }^{60} \mathrm{Co}$} & 1 & $8(66.7)$ & 47.2 & $1.77 \cdot 10^{+3}$ & $3.75 \cdot 10^{+2}$ \\
\hline & 2 & $20(64.5)$ & 14.1 & $1.25 \cdot 10^{+5}$ & $1.63 \cdot 10^{+4}$ \\
\hline & 3 & $15(57.7)$ & 5.37 & $4.53 \cdot 10^{+3}$ & $5.16 \cdot 10^{+2}$ \\
\hline & $1+2+3$ & $43(62.3)$ & 5.37 & $1.25 \cdot 10^{+5}$ & $7.81 \cdot 10^{+3}$ \\
\hline & 4 & $44(80)$ & 62.1 & $1.55 \cdot 10^{+5}$ & $1.52 \cdot 10^{+4}$ \\
\hline
\end{tabular}

Note. ${ }^{\star}$ - in brackets, proportion of measurement results is shown > MDA (in \% of stream samples number). 
Table 4. Features of specific activity of difficult-to-measure radionuclides in SRW streams

\begin{tabular}{|c|c|c|c|c|c|}
\hline \multirow{2}{*}{ Radionuclides } & \multirow{2}{*}{ Stream No. } & \multirow{2}{*}{$\begin{array}{c}\text { Data amount } \\
(>\mathrm{MDA})^{\star}\end{array}$} & \multicolumn{3}{|c|}{ Specific activity, Bq/kg } \\
\hline & & & minimum & maximum & average \\
\hline \multirow{5}{*}{${ }^{94} \mathrm{Nb}$} & 1 & $8(66.7)$ & 7.18 & $4.36 \cdot 10^{+2}$ & $1.16 \cdot 10^{+2}$ \\
\hline & 2 & $17(54.8)$ & 8.82 & $1.50 \cdot 10^{+5}$ & $1.49 \cdot 10^{+4}$ \\
\hline & 3 & $14(53.8)$ & 5.80 & $1.05 \cdot 10^{+4}$ & $8.78 \cdot 10^{+2}$ \\
\hline & $1+2+3$ & $39(56.5)$ & 5.80 & $1.50 \cdot 10^{+5}$ & $6.82 \cdot 10^{+3}$ \\
\hline & 4 & $37(67.3)$ & 17.3 & $3.92 \cdot 10^{+4}$ & $3.97 \cdot 10^{+3}$ \\
\hline \multirow{5}{*}{${ }^{241} \mathrm{Am}$} & 1 & $5(41.7)$ & 13.8 & $1.14 \cdot 10^{+4}$ & $2.43 \cdot 10^{+3}$ \\
\hline & 2 & $11(35.5)$ & 85.0 & $6.35 \cdot 10^{+3}$ & $1.34 \cdot 10^{+3}$ \\
\hline & 3 & $14(53.8)$ & 74.7 & $2.62 \cdot 10^{+3}$ & $4.41 \cdot 10^{+2}$ \\
\hline & $1+2+3$ & $30(43.5)$ & 13.8 & $1.14 \cdot 10^{+4}$ & $1.10 \cdot 10^{+3}$ \\
\hline & 4 & $24(43.6)$ & 44.9 & $1.96 \cdot 10^{+4}$ & $3.00 \cdot 10^{+3}$ \\
\hline \multirow{5}{*}{${ }^{90} \mathrm{Sr}$} & 1 & $3(25)$ & $1.56 \cdot 10^{+2}$ & $1.61 \cdot 10^{+4}$ & $5.49 \cdot 10^{+3}$ \\
\hline & 2 & $21(67.7)$ & 77.0 & $1.13 \cdot 10^{+5}$ & $1.73 \cdot 10^{+4}$ \\
\hline & 3 & $11(42.3)$ & $2.36 \cdot 10^{+2}$ & $1.15 \cdot 10^{+4}$ & $3.91 \cdot 10^{+3}$ \\
\hline & $1+2+3$ & $35(50.7)$ & 77.0 & $1.13 \cdot 10^{+5}$ & $1.21 \cdot 10^{+4}$ \\
\hline & 4 & $53(96.4)$ & 61.5 & $4.34 \cdot 10^{+5}$ & $3.15 \cdot 10^{+4}$ \\
\hline \multirow{5}{*}{${ }^{14} \mathrm{C}$} & 1 & $1(8.3)$ & \multicolumn{3}{|c|}{98.0} \\
\hline & 2 & $7(22.6)$ & $1.07 \cdot 10^{+2}$ & $1.28 \cdot 10^{+2}$ & $1.18 \cdot 10^{+2}$ \\
\hline & 3 & $7(27)$ & 99.0 & $1.32 \cdot 10^{+2}$ & $1.10 \cdot 10^{+2}$ \\
\hline & $1+2+3$ & $15(21.7)$ & 98.0 & $1.32 \cdot 10^{+2}$ & $1.13 \cdot 10^{+2}$ \\
\hline & 4 & $30(54.5)$ & $1.06 \cdot 10^{+2}$ & $1.56 \cdot 10^{+2}$ & $1.35 \cdot 10^{+2}$ \\
\hline \multirow{5}{*}{${ }^{3} \mathrm{H}$} & 1 & $2(16.7)$ & $8.97 \cdot 10^{+2}$ & $1.26 \cdot 10^{+3}$ & $1.08 \cdot 10^{+3}$ \\
\hline & 2 & $14(45.2)$ & $4.65 \cdot 10^{+2}$ & $3.54 \cdot 10^{+3}$ & $1.39 \cdot 10^{+3}$ \\
\hline & 3 & $11(42.3)$ & $5.73 \cdot 10^{+2}$ & $3.01 \cdot 10^{+3}$ & $1.15 \cdot 10^{+3}$ \\
\hline & $1+2+3$ & $27(39.1)$ & $4.65 \cdot 10^{+2}$ & $3.54 \cdot 10^{+3}$ & $1.27 \cdot 10^{+3}$ \\
\hline & 4 & $19(34.5)$ & $5.12 \cdot 10^{+2}$ & $3.72 \cdot 10^{+3}$ & $1.57 \cdot 10^{+3}$ \\
\hline \multirow{5}{*}{${ }^{235} \mathrm{U}$} & 1 & 0 & \multicolumn{3}{|c|}{-} \\
\hline & 2 & $9(29)$ & $2.11 \cdot 10^{-2}$ & $2.78 \cdot 10^{-1}$ & $1.30 \cdot 10^{-1}$ \\
\hline & 3 & $2(7.7)$ & $4.04 \cdot 10^{-2}$ & $8.79 \cdot 10^{-2}$ & $6.42 \cdot 10^{-2}$ \\
\hline & $2+3$ & $11(16)$ & $2.11 \cdot 10^{-2}$ & $2.78 \cdot 10^{-1}$ & $1.18 \cdot 10^{-1}$ \\
\hline & 4 & $23(41.8)$ & $7.92 \cdot 10^{-3}$ & $5.13 \cdot 10^{-1}$ & $1.79 \cdot 10^{-1}$ \\
\hline \multirow{5}{*}{${ }^{238} \mathrm{U}$} & 1 & 0 & \multicolumn{3}{|c|}{-} \\
\hline & 2 & $9(29)$ & $2.50 \cdot 10^{-1}$ & 4.80 & 2.36 \\
\hline & 3 & $2(7.7)$ & $9.55 \cdot 10^{-1}$ & 2.04 & 1.50 \\
\hline & $2+3$ & $11(16)$ & $2.50 \cdot 10^{-1}$ & 4.80 & 2.20 \\
\hline & 4 & $25(45.5)$ & $6.64 \cdot 10^{-2}$ & 7.98 & 2.72 \\
\hline
\end{tabular}

Note. ${ }^{\star}$ - in brackets, proportion of measurement results is shown $>$ MDA (in \% of stream samples number).

specific activity of above listed nuclides can be either higher or lower, than in the eastern compartment;

specific activity of uranium isotopes in noncombustible SRW of western compartment is, generally, lower than in the eastern one, from 2 to 20 times for ${ }^{235} \mathrm{U}$ and from 2 to 4 times for ${ }^{238} \mathrm{U}$. For combustible SRW in the eastern compartment, the lack of available data makes it impossible to carry out a comparative analysis;

specific activity of ${ }^{3} \mathrm{He}$ and ${ }^{14} \mathrm{C}$ nuclides in SRW for all streams in the both compartments is, approximately, at the same level.
The data in Table 5 show the results of regression analysis, assessment of CC values and reliability degree of established correlation relationships between radionuclide activities in the SRW streams. As it can be seen from the data presented, in 24 cases (41\%) only there is a close correlation with reliable CC. Out of 6 datasets with weak relationship of radionuclide activities, two interdependencies only can be considered as reliable ones. In other cases, there is no correlation between radionuclide specific activity.

The illustrations in Figs. 2-6 supplement the data in Table 5 and demonstrate the ranges of scattered values 
Table 5. Results of check of presence/absence of linear correlation between logarithms of radionuclide specific activities in waste contamination spectrum (western "light" compartment)

\begin{tabular}{|c|c|c|c|c|c|c|c|c|}
\hline \multirow[t]{2}{*}{ No. } & \multicolumn{2}{|c|}{ Radionuclides } & \multirow[t]{2}{*}{ Stream No. } & \multirow{2}{*}{$\begin{array}{l}\text { Data amount } \\
\text { (>MDA) }\end{array}$} & \multicolumn{2}{|c|}{ Regression parameters } & \multicolumn{2}{|c|}{$\begin{array}{c}\text { Correlation coefficient (CC) } \\
\text { and its reliability }\end{array}$} \\
\hline & $\mathrm{Y}$ & $\mathrm{X}$ & & & $\operatorname{Ln}(a)$ & $\mathrm{b}$ & $\mathrm{CC}$ & Reliability \\
\hline 1 & ${ }^{137} \mathrm{Cs}$ & ${ }^{60} \mathrm{Co}$ & 1 & 8 & $5.65 \pm 7.81$ & $0.83 \pm 1.44$ & $0.50 \pm 0.18$ & $\mathrm{NC}$ \\
\hline 2 & ${ }^{137} \mathrm{Cs}$ & ${ }^{60} \mathrm{Co}$ & 2 & 20 & $6.91 \pm 1.46$ & $0.43 \pm 0.19$ & $0.75 \pm 0.06$ & $\mathrm{CC}^{+}$ \\
\hline 3 & ${ }^{137} \mathrm{Cs}$ & ${ }^{60} \mathrm{Co}$ & 3 & 15 & $8.00 \pm 2.59$ & $0.20 \pm 0.51$ & $0.23 \pm 0.16$ & $\mathrm{NC}$ \\
\hline 4 & ${ }^{137} \mathrm{Cs}$ & ${ }^{60} \mathrm{Co}$ & $1+2+3$ & 43 & $7.37 \pm 1.11$ & $0.38 \pm 0.17$ & $0.57 \pm 0.07$ & $\mathrm{CC}^{+}$ \\
\hline 5 & ${ }^{137} \mathrm{Cs}$ & ${ }^{60} \mathrm{Co}$ & 4 & 44 & $5.93 \pm 1.42$ & $0.65 \pm 0.18$ & $0.73 \pm 0.05$ & $\mathrm{CC}^{+}$ \\
\hline 6 & ${ }^{90} \mathrm{Sr}$ & ${ }^{137} \mathrm{Cs}$ & 1 & 3 & $-26.32 \pm 6.15$ & $4.87 \pm 90.79$ & $0.56 \pm 0.26$ & WC / CC- \\
\hline 7 & ${ }^{90} \mathrm{Sr}$ & ${ }^{137} \mathrm{Cs}$ & 2 & 21 & $0.84 \pm 2.40$ & $0.80 \pm 0.24$ & $0.85 \pm 0.04$ & $\mathrm{CC}^{+}$ \\
\hline 8 & ${ }^{90} \mathrm{Sr}$ & ${ }^{137} \mathrm{Cs}$ & 3 & 11 & $3.37 \pm 5.19$ & $0.46 \pm 0.53$ & $0.55 \pm 0.14$ & $\mathrm{WC} / \mathrm{CC}^{-}$ \\
\hline 9 & ${ }^{90} \mathrm{Sr}$ & ${ }^{137} \mathrm{Cs}$ & $1+2+3$ & 35 & $1.47 \pm 2.10$ & $0.72 \pm 0.22$ & $0.75 \pm 0.05$ & $\mathrm{CC}^{+}$ \\
\hline 10 & ${ }^{90} \mathrm{Sr}$ & ${ }^{137} \mathrm{Cs}$ & 4 & 53 & $2.54 \pm 1.91$ & $0.62 \pm 0.18$ & $0.68 \pm 0.05$ & $\mathrm{CC}^{+}$ \\
\hline 11 & ${ }^{90} \mathrm{Sr}$ & ${ }^{60} \mathrm{Co}$ & 2 & 16 & $7.70 \pm 2.40$ & $0.21 \pm 0.28$ & $0.39 \pm 0.14$ & $\mathrm{NC}$ \\
\hline 12 & ${ }^{90} \mathrm{Sr}$ & ${ }^{60} \mathrm{Co}$ & 3 & 7 & $9.25 \pm 2.63$ & $-0.21 \pm 0.45$ & $0.48 \pm 0.20$ & $\mathrm{NC}$ \\
\hline 13 & ${ }^{90} \mathrm{Sr}$ & ${ }^{60} \mathrm{Co}$ & $2+3$ & 23 & $7.22 \pm 1.65$ & $0.24 \pm 0.21$ & $0.46 \pm 0.11$ & $\mathrm{WC} / \mathrm{CC}^{-}$ \\
\hline 14 & ${ }^{90} \mathrm{Sr}$ & ${ }^{60} \mathrm{Co}$ & 4 & 43 & $6.92 \pm 1.64$ & $0.30 \pm 0.21$ & $0.40 \pm 0.09$ & $\mathrm{WC} / \mathrm{CC}^{+}$ \\
\hline 15 & ${ }^{94} \mathrm{Nb}$ & ${ }^{60} \mathrm{Co}$ & 1 & 8 & $-1.21 \pm 6.07$ & $0.95 \pm 1.12$ & $0.65 \pm 0.19$ & $\mathrm{WC} / \mathrm{CC}^{+}$ \\
\hline 16 & ${ }^{94} \mathrm{Nb}$ & ${ }^{60} \mathrm{Co}$ & 2 & 15 & $-0.41 \pm 1.65$ & $0.95 \pm 0.20$ & $0.95 \pm 0.02$ & $\mathrm{CC}^{+}$ \\
\hline 17 & ${ }^{94} \mathrm{Nb}$ & ${ }^{60} \mathrm{Co}$ & 3 & 13 & $-0.29 \pm 1.90$ & $0.96 \pm 0.35$ & $0.88 \pm 0.04$ & $\mathrm{CC}^{+}$ \\
\hline 18 & ${ }^{94} \mathrm{Nb}$ & ${ }^{60} \mathrm{Co}$ & $1+2+3$ & 36 & $-0.62 \pm 0.93$ & $0.96 \pm 0.14$ & $0.92 \pm 0.02$ & $\mathrm{CC}^{+}$ \\
\hline 19 & ${ }^{94} \mathrm{Nb}$ & ${ }^{60} \mathrm{Co}$ & 4 & 34 & $0.46 \pm 1.41$ & $0.78 \pm 0.17$ & $0.85 \pm 0.03$ & $\mathrm{CC}^{+}$ \\
\hline 20 & ${ }^{94} \mathrm{Nb}$ & ${ }^{137} \mathrm{Cs}$ & 1 & 8 & $-1.82 \pm 6.95$ & $0.56 \pm 0.68$ & $0.64 \pm 0.14$ & $\mathrm{CC}^{+}$ \\
\hline 21 & ${ }^{94} \mathrm{Nb}$ & ${ }^{137} \mathrm{Cs}$ & 2 & 17 & $-4.58 \pm 8.46$ & $1.10 \pm 0.82$ & $0.59 \pm 0.11$ & $\mathrm{CC}^{+}$ \\
\hline 22 & ${ }^{94} \mathrm{Nb}$ & ${ }^{137} \mathrm{Cs}$ & 3 & 14 & $2.25 \pm 7.21$ & $0.25 \pm 0.81$ & $0.19 \pm 0.17$ & $\mathrm{NC}$ \\
\hline 23 & ${ }^{94} \mathrm{Nb}$ & ${ }^{137} \mathrm{Cs}$ & $1+2+3$ & 39 & $-2.01 \pm 4.33$ & $0.75 \pm 0.44$ & $0.48 \pm 0.08$ & $\mathrm{CC}^{+}$ \\
\hline 24 & ${ }^{94} \mathrm{Nb}$ & ${ }^{137} \mathrm{Cs}$ & 4 & 37 & $0.44 \pm 3.10$ & $0.57 \pm 0.28$ & $0.56 \pm 0.08$ & $\mathrm{CC}^{+}$ \\
\hline 25 & ${ }^{235} \mathrm{U}$ & ${ }^{137} \mathrm{Cs}$ & 2 & 9 & $-5.68 \pm 8.12$ & $0.29 \pm 0.73$ & $0.33 \pm 0.20$ & $\mathrm{NC}$ \\
\hline 26 & ${ }^{235} \mathrm{U}$ & ${ }^{137} \mathrm{Cs}$ & $2+3$ & 11 & $-5.44 \pm 6.71$ & $0.26 \pm 0.61$ & $0.31 \pm 0.18$ & $\mathrm{NC}$ \\
\hline 27 & ${ }^{235} \mathrm{U}$ & ${ }^{137} \mathrm{Cs}$ & 4 & 23 & $-6.01 \pm 2.83$ & $0.33 \pm 0.24$ & $0.52 \pm 0.10$ & $\mathrm{CC}^{+}$ \\
\hline 28 & ${ }^{238} \mathrm{U}$ & ${ }^{137} \mathrm{Cs}$ & 2 & 9 & $-3.69 \pm 7.79$ & $0.37 \pm 0.70$ & $0.43 \pm 0.18$ & $\mathrm{NC}$ \\
\hline 29 & ${ }^{238} \mathrm{U}$ & ${ }^{137} \mathrm{Cs}$ & $2+3$ & 11 & $-3.13 \pm 6.47$ & $0.32 \pm 0.59$ & $0.38 \pm 0.17$ & $\mathrm{NC}$ \\
\hline 30 & ${ }^{238} \mathrm{U}$ & ${ }^{137} \mathrm{Cs}$ & 4 & 25 & $-2.71 \pm 3.14$ & $0.28 \pm 0.27$ & $0.40 \pm 0.11$ & $\mathrm{WC} / \mathrm{CC}^{-}$ \\
\hline 31 & ${ }^{235} \mathrm{U}$ & ${ }^{60} \mathrm{Co}$ & 2 & 8 & $-1.09 \pm 3.77$ & $-0.18 \pm 0.43$ & $0.38 \pm 0.20$ & $\mathrm{NC}$ \\
\hline 32 & ${ }^{235} \mathrm{U}$ & ${ }^{60} \mathrm{Co}$ & $2+3$ & 10 & $-1.77 \pm 2.87$ & $-0.11 \pm 0.34$ & $0.25 \pm 0.20$ & $\mathrm{NC}$ \\
\hline 33 & ${ }^{235} \mathrm{U}$ & ${ }^{60} \mathrm{Co}$ & 4 & 20 & $-3.05 \pm 2.43$ & $0.10 \pm 0.28$ & $0.18 \pm 0.14$ & $\mathrm{NC}$ \\
\hline 34 & ${ }^{238} \mathrm{U}$ & ${ }^{60} \mathrm{Co}$ & 2 & 8 & $1.75 \pm 3.77$ & $-0.17 \pm 0.43$ & $0.37 \pm 0.20$ & $\mathrm{NC}$ \\
\hline 35 & ${ }^{238} \mathrm{U}$ & ${ }^{60} \mathrm{Co}$ & $2-3$ & 10 & $1.25 \pm 2.83$ & $-0.11 \pm 0.33$ & $0.27 \pm 0.20$ & $\mathrm{NC}$ \\
\hline 36 & ${ }^{238} \mathrm{U}$ & ${ }^{60} \mathrm{Co}$ & 4 & 22 & $-0.60 \pm 2.60$ & $0.12 \pm 0.30$ & $0.19 \pm 0.14$ & $\mathrm{NC}$ \\
\hline 37 & ${ }^{241} \mathrm{Am}$ & ${ }^{137} \mathrm{Cs}$ & 1 & 5 & $-7.61 \pm 19.72$ & $1.23 \pm 1.83$ & $0.78 \pm 0.12$ & $\mathrm{CC}^{+}$ \\
\hline 38 & ${ }^{241} \mathrm{Am}$ & ${ }^{137} \mathrm{Cs}$ & 2 & 11 & $-0.85 \pm 6.15$ & $0.71 \pm 0.61$ & $0.66 \pm 0.11$ & $\mathrm{CC}^{+}$ \\
\hline
\end{tabular}


Table 5. Results of check of presence/absence of linear correlation between logarithms of radionuclide specific activities in waste contamination spectrum (western "light" compartment), Continued

\begin{tabular}{|c|c|c|c|c|c|c|c|c|}
\hline \multirow[t]{2}{*}{ No. } & \multicolumn{2}{|c|}{ Radionuclides } & \multirow[t]{2}{*}{ Stream No. } & \multirow{2}{*}{$\begin{array}{l}\text { Data amount } \\
\text { (>MDA) }\end{array}$} & \multicolumn{2}{|c|}{ Regression parameters } & \multicolumn{2}{|c|}{$\begin{array}{c}\text { Correlation coefficient (CC) } \\
\text { and its reliability }\end{array}$} \\
\hline & $\mathrm{Y}$ & $\mathrm{X}$ & & & $\operatorname{Ln}(\mathrm{a})$ & $\mathrm{b}$ & $\mathrm{CC}$ & Reliability \\
\hline 39 & ${ }^{241} \mathrm{Am}$ & ${ }^{137} \mathrm{Cs}$ & 3 & 14 & $-0.71 \pm 4.75$ & $0.67 \pm 0.51$ & $0.64 \pm 0.11$ & $\mathrm{CC}^{+}$ \\
\hline 40 & ${ }^{241} \mathrm{Am}$ & ${ }^{137} \mathrm{Cs}$ & $1+2+3$ & 30 & $-1.26 \pm 3.29$ & $0.72 \pm 0.33$ & $0.64 \pm 0.07$ & $\mathrm{CC}^{+}$ \\
\hline 41 & ${ }^{241} \mathrm{Am}$ & ${ }^{137} \mathrm{Cs}$ & 4 & 24 & $0.31 \pm 2.63$ & $0.61 \pm 0.23$ & $0.76 \pm 0.06$ & $\mathrm{CC}^{+}$ \\
\hline 42 & ${ }^{241} \mathrm{Am}$ & ${ }^{60} \mathrm{Co}$ & 1 & 5 & $12.48 \pm 55.75$ & $-1.31 \pm 10.59$ & $0.05 \pm 0.22$ & $\mathrm{NC}$ \\
\hline 43 & ${ }^{241} \mathrm{Am}$ & ${ }^{60} \mathrm{Co}$ & 2 & 10 & $5.51 \pm 3.67$ & $0.10 \pm 0.49$ & $0.17 \pm 0.21$ & $\mathrm{NC}$ \\
\hline 44 & ${ }^{241} \mathrm{Am}$ & ${ }^{60} \mathrm{Co}$ & 3 & 9 & $4.22 \pm 2.31$ & $0.27 \pm 0.49$ & $0.45 \pm 0.18$ & $\mathrm{NC}$ \\
\hline 45 & ${ }^{241} \mathrm{Am}$ & ${ }^{60} \mathrm{Co}$ & $1+2+3$ & 24 & $4.73 \pm 1.82$ & $0.19 \pm 0.30$ & $0.19 \pm 0.13$ & $\mathrm{NC}$ \\
\hline 46 & ${ }^{241} \mathrm{Am}$ & ${ }^{60} \mathrm{Co}$ & 4 & 21 & $4.42 \pm 1.73$ & $0.38 \pm 0.22$ & $0.63 \pm 0.09$ & $\mathrm{CC}^{+}$ \\
\hline 47 & ${ }^{3} \mathrm{He}$ & ${ }^{60} \mathrm{Co}$ & $1+2+3$ & 20 & $6.78 \pm 0.82$ & $0.04 \pm 0.11$ & $0.18 \pm 0.14$ & $\mathrm{NC}$ \\
\hline 48 & ${ }^{3} \mathrm{He}$ & ${ }^{60} \mathrm{Co}$ & 4 & 17 & $7.44 \pm 1.10$ & $-0.03 \pm 0.12$ & $0.13 \pm 0.16$ & $\mathrm{NC}$ \\
\hline 49 & ${ }^{3} \mathrm{He}$ & ${ }^{137} \mathrm{Cs}$ & $1+2+3$ & 27 & $7.32 \pm 1.69$ & $0.04 \pm 0.16$ & $0.09 \pm 0.13$ & $\mathrm{NC}$ \\
\hline 50 & ${ }^{3} \mathrm{He}$ & ${ }^{137} \mathrm{Cs}$ & 4 & 19 & $8.02 \pm 1.13$ & $-0.08 \pm 0.10$ & $0.35 \pm 0.13$ & $\mathrm{NC}$ \\
\hline 51 & ${ }^{14} \mathrm{C}$ & ${ }^{60} \mathrm{Co}$ & $1+2+3$ & 13 & $4.70 \pm 0.22$ & $0.00 \pm 0.03$ & $0.05 \pm 0.19$ & $\mathrm{NC}$ \\
\hline 52 & ${ }^{14} \mathrm{C}$ & ${ }^{60} \mathrm{Co}$ & 4 & 27 & $4.89 \pm 0.16$ & $0.00 \pm 0.02$ & $0.05 \pm 0.19$ & $\mathrm{NC}$ \\
\hline 53 & ${ }^{14} \mathrm{C}$ & ${ }^{137} \mathrm{Cs}$ & $1+2+3$ & 15 & $4.69 \pm 0.57$ & $0.00 \pm 0.05$ & $0.03 \pm 0.17$ & $\mathrm{NC}$ \\
\hline 54 & ${ }^{14} \mathrm{C}$ & ${ }^{137} \mathrm{Cs}$ & 4 & 30 & $4.97 \pm 0.25$ & $-0.01 \pm 0.02$ & $0.12 \pm 0.12$ & $\mathrm{NC}$ \\
\hline 55 & ${ }^{235} \mathrm{U}$ & ${ }^{238} \mathrm{U}$ & 2 & 9 & $-2.91 \pm 0.23$ & $0.97 \pm 0.20$ & $0.97 \pm 0.01$ & $\mathrm{CC}^{+}$ \\
\hline 56 & ${ }^{235} \mathrm{U}$ & ${ }^{238} \mathrm{U}$ & $2+3$ & 11 & $-2.96 \pm 0.19$ & $0.98 \pm 0.18$ & $0.97 \pm 0.01$ & $\mathrm{CC}^{+}$ \\
\hline 57 & ${ }^{235} \mathrm{U}$ & ${ }^{238} \mathrm{U}$ & 4 & 23 & $-3.07 \pm 0.21$ & $1.19 \pm 0.18$ & $0.95 \pm 0.01$ & $\mathrm{CC}^{+}$ \\
\hline 58 & ${ }^{14} \mathrm{C}$ & ${ }^{3} \mathrm{He}$ & $2+3$ & 12 & $5.01 \pm 0.18$ & $-0.04 \pm 0.11$ & $0.24 \pm 0.18$ & $\mathrm{NC}$ \\
\hline 59 & ${ }^{14} \mathrm{C}$ & ${ }^{3} \mathrm{He}$ & 4 & 15 & $4.57 \pm 0.48$ & $0.05 \pm 0.07$ & $0.42 \pm 0.14$ & $\mathrm{NC}$ \\
\hline
\end{tabular}

Notes. For parameters of logarithm linear regression and CC value, 95\% confidence interval for estimating the mean value is given. Designations in the column "Reliability": $\mathrm{CC}^{+}$- correlation is present, correlation coefficient is reliable; NC - no correlation; $\mathrm{WC} / \mathrm{CC}^{+}-$correlation is weak, but correlation coefficient is quite reliable; $\mathrm{WC} / \mathrm{CC}^{-}-$correlation is weak and correlation coefficient is unreliable.

of radionuclide specific activities (data "cloud") in stream samples of measurement results with the highest $\mathrm{CC}$ value. According to their form, data "clouds" shown in the figures have the same typical features as it was earlier highlighted for the eastern compartment.

For DTM nuclide - KN pairs, the measurement results were analyzed with taking into account the probability of having two alternative KNs for one DTM nuclide. International experience in solving the same problems recommends making the choice of the most acceptable $\mathrm{KN}$, based not on the expected similarity of the mechanisms of formation and physicochemical characteristics of both nuclides, but solely on the presence or absence of a correlation between the measured activities of radionuclides in waste materials. During the operation of one or another type of NPP power Unit, as a result of radioactivity transfer through the technological channels in reactor core, its distribution in various rooms and, ultimately, deposition on various materials, forms those typical features that are, subsequently, found in the liquid and solid waste sent for disposal. The examples of such situations reflected in Table 5 are the correlations established for individual SRW streams between ${ }^{90} \mathrm{Sr}-{ }^{60} \mathrm{Co}$ (items 14), ${ }^{94} \mathrm{Nb}-{ }^{137} \mathrm{Cs}$ (items 20, 21, 23, and 24), and ${ }^{241} \mathrm{Am}-$ ${ }^{60} \mathrm{Co}$ (items 46), and, conversely, their absence where they, theoretically, should be present in accordance with the known mechanisms of their formation and transport, namely, between ${ }^{90} \mathrm{Sr}-{ }^{137} \mathrm{Cs}$ (items 6, 8), ${ }^{235} \mathrm{U} /{ }^{238} \mathrm{U}-{ }^{137} \mathrm{Cs}$ (items 25,26 and $28,29,30$ ) and ${ }^{3} \mathrm{He} /{ }^{14} \mathrm{C}-{ }^{60} \mathrm{Co}$ (items 47,48 and 51, 52). 


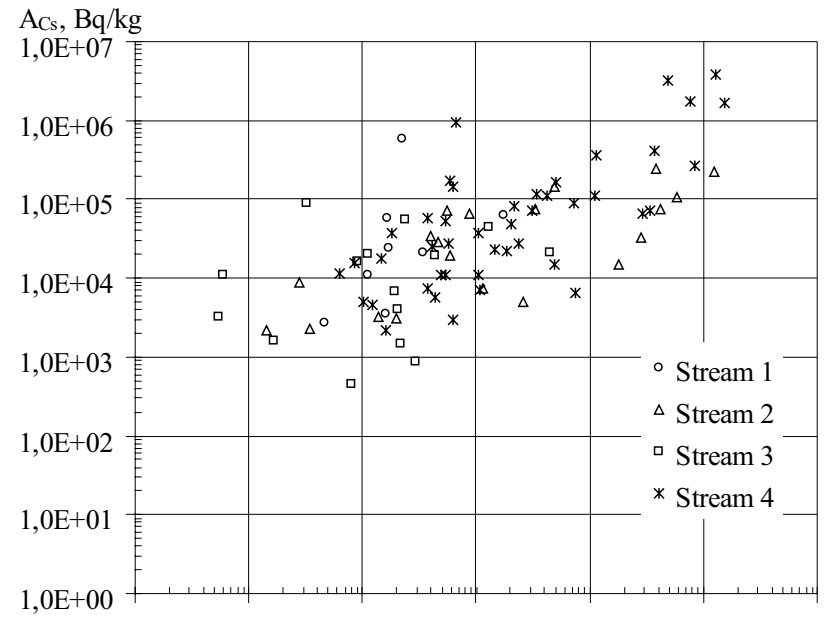

$1,0 \mathrm{E}+00 \quad 1,0 \mathrm{E}+01 \quad 1,0 \mathrm{E}+02 \quad 1,0 \mathrm{E}+03 \quad 1,0 \mathrm{E}+04 \quad 1,0 \mathrm{E}+05 \quad 1,0 \mathrm{E}+06$

Fig. 2. Specific activity of ${ }^{137} \mathrm{Cs}$ and ${ }^{60} \mathrm{Co}$ in SRW

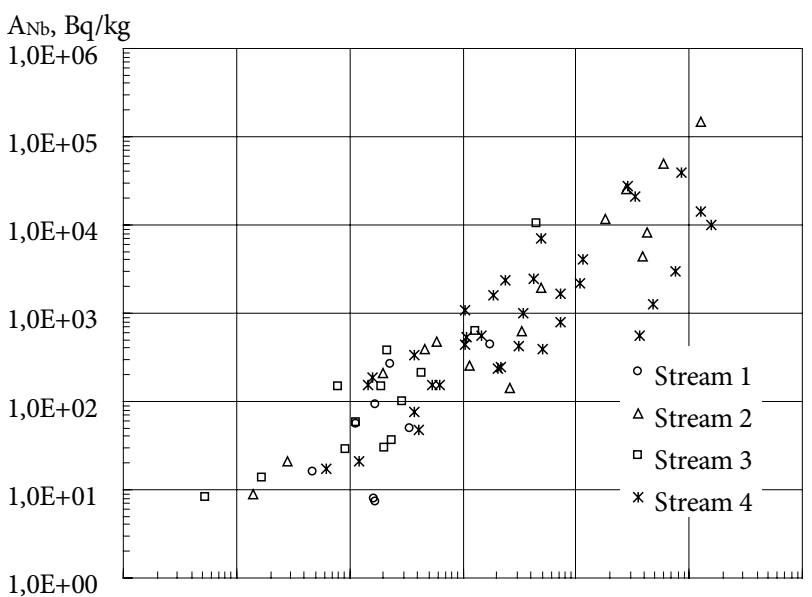

$1,0 \mathrm{E}+00 \quad 1,0 \mathrm{E}+01 \quad 1,0 \mathrm{E}+02 \quad 1,0 \mathrm{E}+03 \quad 1,0 \mathrm{E}+04 \quad 1,0 \mathrm{E}+05 \quad 1,0 \mathrm{E}+06$ $\mathrm{Aco}_{\mathrm{C}} \mathrm{Bq} / \mathrm{kg}$

Fig. 4. Specific activity of ${ }^{94} \mathrm{Nb}$ and ${ }^{60} \mathrm{Co}$ in SRW

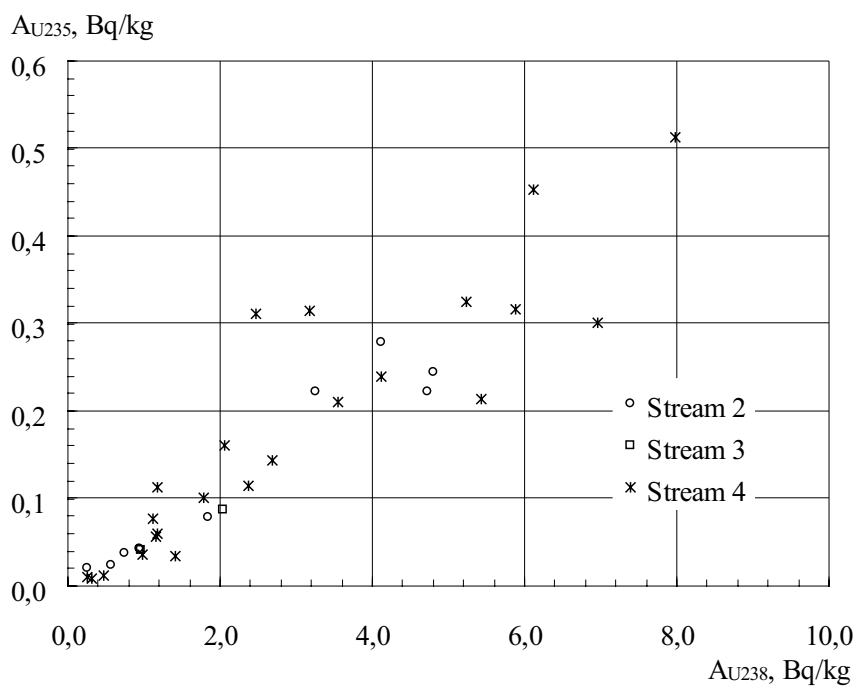

Fig. 6. Specific activity of ${ }^{235} \mathrm{U}$ and ${ }^{238} \mathrm{U}$ in SRW

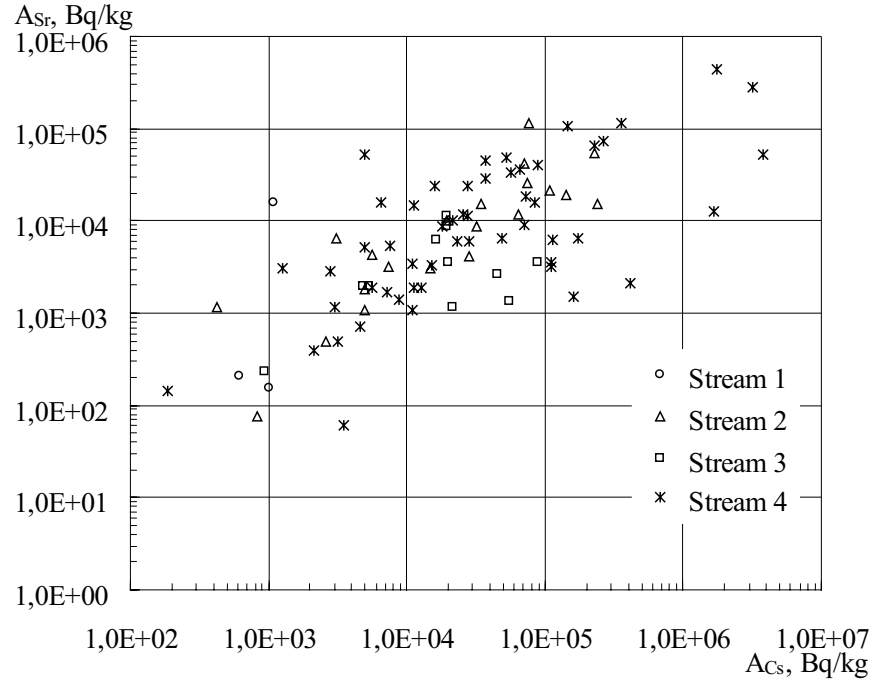

Fig. 3. Specific activity of ${ }^{90} \mathrm{Sr}$ and ${ }^{137} \mathrm{Cs}$ in SRW

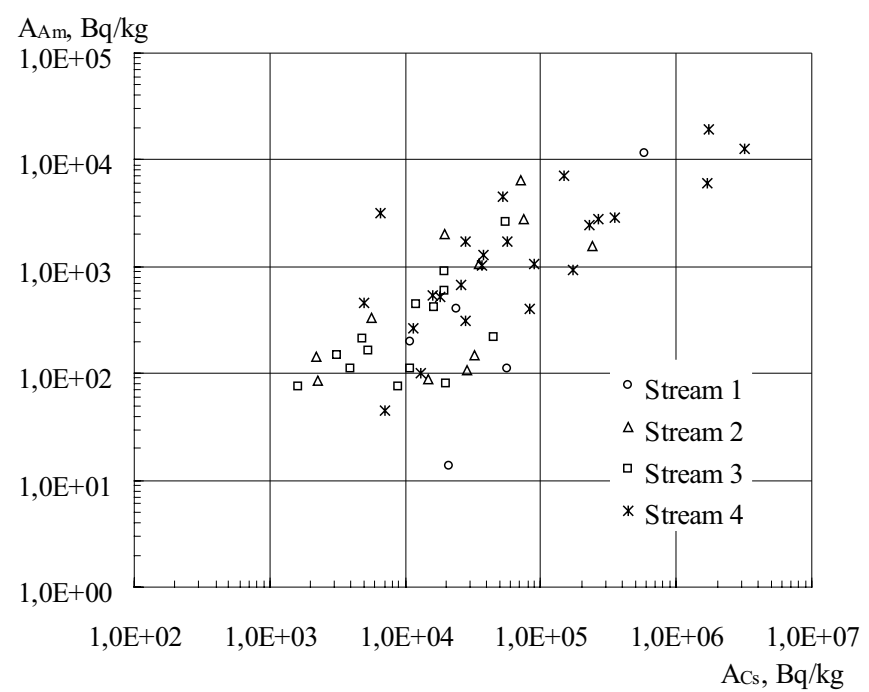

Fig. 5. Specific activity of ${ }^{241} \mathrm{Am}$ and ${ }^{137} \mathrm{Cs}$ in SRW

Thus, if we summarize the discussion of made results of correlation - regression analysis, one can conclude that the regression dependences established in one third part of datasets of measurement results only can be considered as satisfactory for DTM nuclide activity certification in the SRW. At the same time, the data obtained give ground to consider that the combination of stream samples can significantly improve the statistical reliability of estimated activity of DTM nuclides, such as ${ }^{90} \mathrm{Sr}$ and ${ }^{94} \mathrm{Nb}$ in non-combustible SRW (see Table 5, items 14, 23) using the established functional dependencies. It should be noted, when evaluating the data for SRW in eastern compartment [7], such a methodological technique provided essential improvement in plausibility of certification results for a number of DTM nuclides, with considering a small number of samples with specific activity above the MDA 
in individual waste streams. The results of investigated SRW, which are being stored in RSRW western compartment, confirm, in general, previously obtained data [7] indicating the fact that the specific activity of huge amount of nuclides subject to certification, is below the MDA. For ${ }^{137} \mathrm{Cs},{ }^{60} \mathrm{Co},{ }^{90} \mathrm{Sr},{ }^{94} \mathrm{Nb},{ }^{241} \mathrm{Am},{ }^{14} \mathrm{C},{ }^{3} \mathrm{H}$ and ${ }^{235,238} \mathrm{U}$ measurement results, the established functional dependencies are, in many respects, similar to the results obtained for RSRW eastern compartment, including the fact that there are no relationships for a number of nuclides. This gives grounds to believe that the levels of specific activity of investigated radionuclides and their ratios in the spectrum of radioactive contamination of SRW observable in both RSRW compartments are typical for the ChNPP operational SRW, which had been produced during the period of normal operation of power Units until 1986.

\section{Conclusions}

The experimental data obtained in current work and the results of their statistical analysis allowed assessing typical features of radioactive contamination of new batch of the ChNPP operational SRW, which are planned to transport soon for the burial from RSRW western compartment. Radionuclide content of studied waste materials, like in the neighboring eastern compartment, is fully determined by such nuclides as ${ }^{137} \mathrm{Cs},{ }^{60} \mathrm{Co},{ }^{90} \mathrm{Sr},{ }^{94} \mathrm{Nb},{ }^{241} \mathrm{Am},{ }^{14} \mathrm{C},{ }^{3} \mathrm{H}$, ${ }^{235,238} \mathrm{U}$. Contamination levels of SRW by the rest radionuclides from those, subject to compulsory certification, do not exceed their detection limit (<MDA). For the most important measurement results, functional dependencies between radionuclide activities in contamination spectrum of waste materials in western compartment in many respects reflect the same trends and ratios, which were earlier established for RSRW eastern compartment, including the facts of absence of any interrelations for a number of nuclides.

The data obtained give a ground to expect that the integrating of stream samples could essentially improve the statistical reliability of evaluable certified activity of such DTMs as ${ }^{90} \mathrm{Sr}$ and ${ }^{94} \mathrm{Nb}$ for non-combustible SRW in activity of $\mathrm{KN}\left({ }^{137} \mathrm{Cs}\right.$ and/or $\left.{ }^{60} \mathrm{Co}\right)$ using established functional dependencies.

The data gained in this work allow passing to the next stage of our investigation - evaluation of scaling factors and establishment of radionuclide vectors, whose absence does not allow certifying the DTM nuclides at ICSRWM in SRW packages being transported to the burial from the ChNPP RSRW.

\section{Acknowledgment}

The authors wish to thank Valentyn Bezmylov for participation in the preparation of English version, which presents the results obtained in this work.

\section{References}

1. Criteria for acceptance of waste for burial in specially equipped near-surface repository for solid radwaste (SESRSRW). First stage of SESRSRW operation. Acceptance of RAW from SSE "ChNPP” PTLRW and PTSRW for burial in two symmetrical compartments of SESRSRW. Revision 5. Endorsed by acting Director General of State Corporation “UkrSE 'Radon"”. Chornobyl, 2009. 38 p. (in Ukr.)

2. Decision on conducting the 3rd stage of "hot" tests and the initial stage of industrial operation of the ICMSRW. Conclusion of the State examination of nuclear and radiation safety of the SSE ChNPP documentation. Approved by T. P. Kilochytska, Deputy Chairman of State Nuclear Regulatory Inspectorate of Ukraine (SSE "ChNPP", Archive inv. No. 28 dated July 28, 2016). (in Ukr.)

3. RAW management, radiation safety. Chornobyl NPP (September 12-16, 2016). Report of Technical Support Mission. Moscow: WANO-MC, 2016, 10 p. (TSM43-2016) (in Russ.)

4. Methodology and practice in defining nuclide vectors. Final report of WANO-MC Technical Support Mission at Chernobyl NPP (Slavutych, May 14-18, 2018). Slavutych, 2018, 12 p. (in Russ.)

5. IAEA (2007). Strategy and methodology for radioactive waste characterization. IAEA-TECDOC-1537. Vienna: IAEA, $182 \mathrm{p}$.

6. IAEA (2009). Determination and use of scaling factors for waste characterization in NPP. IAEA Nuclear Energy Series NW-T-1.18. Vienna: IAEA, $142 \mathrm{p}$.

7. Mikhailov A. V., Pavliuchenko N. I., Miasnikov A. V., Terzi A. K. (2019). [Results of radionuclide vectors determination to be used in characterization of SSE NPP's solid radwaste]. Problems of Chornobyl Exclusion Zone, vol. 20, pp. 13-26. (in Russ.)

8. Mykhailov O. V., Bezmylov V. M., Terzi A. K. (2020). Analysis of radionuclide contamination features in solid radwaste of "light" eastern compartment of solid waste repository of Chornobyl NPP. Nuclear Power and the Environment, vol. 16, no. 1, pp. 40-48.

9. Grubbs F. E. (1969). Procedures for detecting outlying observations in samples. Technometrics, vol. 11, no. 1, pp. 1-21.

10. Ross S. M. (2004). Introduction to probability and statistics for engineers and scientists. Third Edition. USA: Elsevier Academic Press, $641 \mathrm{p}$. 


\author{
О. В. Михайлов ${ }^{1}$, А. К. Терзі ${ }^{2}$ \\ ${ }^{1}$ Iнститут проблем безпеки АЕС НАН України, \\ вул. Кірова, 36а, Чорнобиль, 07270, Украӥна \\ 2 ДСП “Чорнобильська АЕС", \\ Славутич, Київська обл., 07101, Україна
}

\section{Дослідження радіонуклідного забруднення матеріалів, що зберігаються у сховищі твердих радіоактивних відходів Чорнобильської АЕС}

У 2016 р. були розпочаті підготовчі роботи для проведення характеризації твердих радіоактивних відходів (ТРВ), що тимчасово зберігалися у сховищі ТРВ ДСП “Чорнобильська АЕС”. За результатами “гарячих" випробувань промислового комплексу із поводження з ТРВ (ПКПТРВ) було виявлено, що безпосередньо в упаковці штатне устаткування системи радіаційно-технологічного контролю дозволяє надійно вимірювати тільки питому активність гамма-випромінювачів ${ }^{60} \mathrm{Co} \mathrm{i}^{137} \mathrm{Cs}$ у відходах. Рішення проблеми було запропоновано виходячи 3 міжнародного досвіду: визначати вміст інших альфа- і бета-випромінюючих нуклідів, що підлягають паспортизації у ТРВ, за методологією радіонуклідних векторів (масштабних коефіцієнтів - scaling factors). Однак це вимагає дослідження кожної окремої партії відходів, проведення вибіркового лабораторного контролю представницьких проб матеріалів і встановлення функціональних взаємозв'язків між вмістом радіонуклідів у зразках за результатами вимірювань.

Метою роботи було провести дослідницькі роботи, розпочаті в “легкому” східному відсіку сховища, й отримати необхідні дані для характеризації ТРВ у сусідньому західному відсіку. Репрезентативні проби матеріалів було відібрано у дев’яти точках відсіку з глибини до 1 м і розділено після візуального огля- ду на чотири потоки відходів. Визначення питомої активності радіонуклідів у зразках ТРВ проводили у вимірювальній водно-радіохімічній лабораторії ЧАЕС з використанням методів альфа-, бета- і гаммаспектрометрії, рідинної сцинтиляції та ІСРМ-спектрометрії. Результати вимірювань було проаналізовано за допомогою кореляційно-регресійного аналізу після відсіву анормальних результатів вимірювань за тестом Граббса. Результати вимірювань у вигляді $<$ МДА (менше значення мінімально детектованої активності) із вибірок даних виключались.

Встановлено, що рівні радіоактивності ТРВ у верхньому шарі “легкого” західного відсіку сховища, як і в сусідньому східному відсіку, визначаються вмістом нуклідів ${ }^{137} \mathrm{Cs},{ }^{60} \mathrm{Co},{ }^{90} \mathrm{Sr},{ }^{94} \mathrm{Nb},{ }^{235} \mathrm{U},{ }^{238} \mathrm{U},{ }^{241} \mathrm{Am}$, ${ }^{3} \mathrm{H}$ та ${ }^{14} \mathrm{C}$. Особливості спектру радіонуклідного забруднення ТРВ в обох відсіках багато в чому схожі, що, найбільш ймовірно, відображає характерні властивості експлуатаційних відходів Чорнобильської AEC, які утворилися в період нормальної роботи енергоблоків станції з 1978 до 1986 р. і були складовані з консервацією в сховищі ще до аварії на 4-му енергоблоці. Однак, порівняно зі східним відсіком, рівні забруднення ТРВ в західному відсіку для таких радіонуклідів, як ${ }^{60} \mathrm{Co},{ }^{94} \mathrm{Nb}$ та ${ }^{90} \mathrm{Sr} €$ істотно вищими, а для ізотопів урану ${ }^{235,238} \mathrm{U}$ навпаки — в кілька разів нижчими. Зроблено висновок про те, що отримані експериментальні та аналітичні результати можуть бути використані як вихідні дані для наступного етапу досліджень - розрахунку масштабних коефіцієнтів і встановлення радіонуклідних векторів, яких не вистачає для проведення паспортизації радіонуклідного складу нової партії ТРВ ЧАЕС, які планується захоронити.

Ключові слова: Чорнобильська АЕС, тверді радіоактивні відходи, питома активність, радіонукліди, що важко вимірюються, реперні радіонукліди, коефіцієнт кореляції, коефіцієнти масштабування.

Надійшла 06.09.2021 Received 06.09.2021 